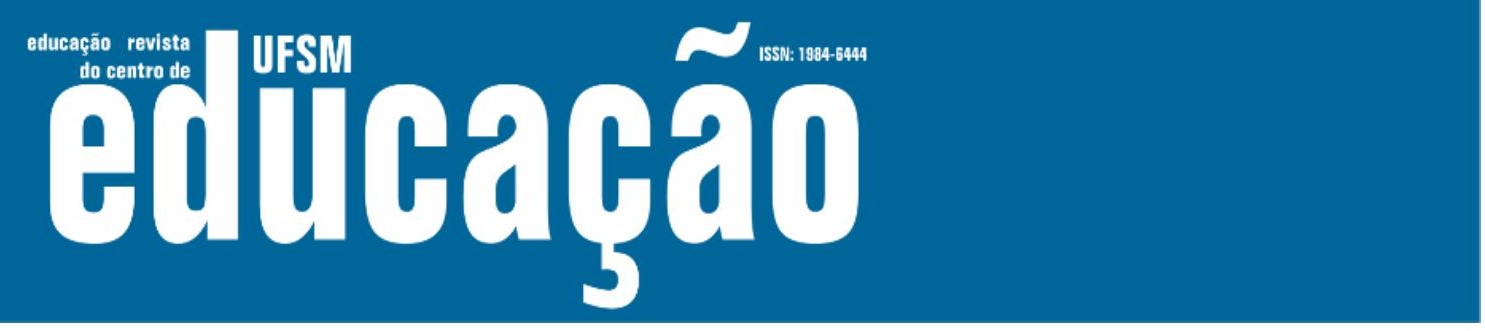

ISSN: 1984-6444 | http://dx.doi.org/10.5902/1984644461431

\title{
Aperçu du champ de la didactique des sciences humaines et sociales au primaire après la parution du rapport de la Commission Parent en 1964 au Québec
}

\author{
Overview of the didactics of humanities and social sciences in \\ elementary school after the 1964 release of the Parent Commission \\ report in Quebec \\ Retrato do campo da didática das ciências humanas no ensino \\ fundamenta (primário) após a publicação do relatório da Comission \\ Parent em 1964 no Quebec
}

\section{Anderson Araújo-Oliveira}

Professor Doutor na Université du Québec à Montréal. Montreal, Quebec, Canadá. araujo-oliveira.anderson@uqam.ca - https://orcid.org/0000-0002-7214-0032

Recebido em 13 de outubro de 2020

Aprovado em 17 de novembro de 2020

Publicado em 31 de julho de 2021

\section{RESUMÉ}

L'article présente une analyse préliminaire de la documentation scientifique québécoise portant sur l'enseignement des sciences humaines et sociales au primaire publiée après la parution du Rapport de la Commission royale d'enquête sur l'enseignement en 1964. Pour se faire, 83 documents publiés depuis près d'une soixantaine d'années ont été revus par l'entremise d'une analyse thématique mixte de contenu en fonction des questionnements et du cadre conceptuel à la base des travaux de recherche antérieurs réalisés par l'auteur du texte. II met ainsi en évidence les tendances, perspectives, tensions et points de rupture concernant les fondements et la trame conceptuelle propres au champ de la didactique des sciences humaines et sociales au primaire au Québec. Influencée tantôt par des théories dites personnalistes et celles dites technologiques, tantôt par des théories dites constructivistes (voire socioconstructivistes), l'évolution de ce champ témoigne d'une diversité de conceptions et de points de vue directement liés aux fondements sousjacents à la pensée de ceux qui collectivement et historiquement la mettent en œuvre. Mots-clés: Didactique; Sciences humaines et sociales; Primaire; Québec. 


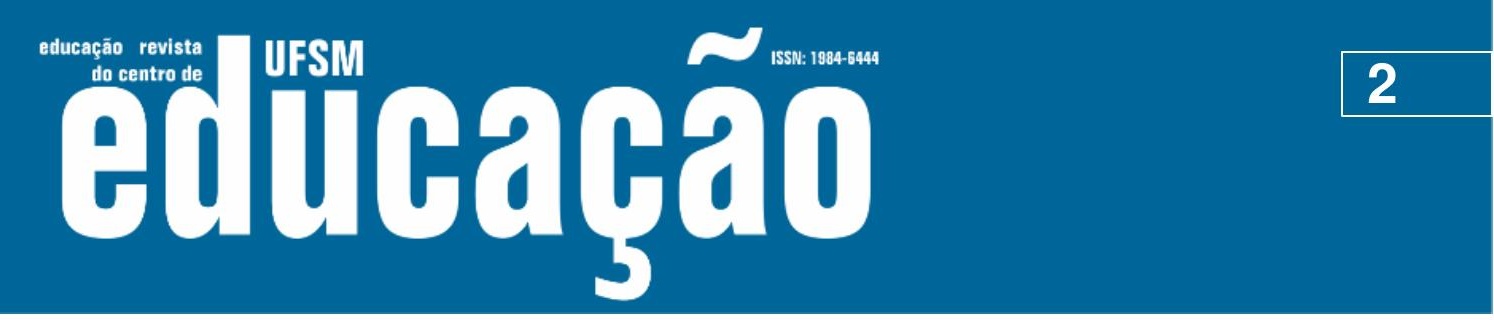

ISSN: 1984-6444 | http://dx.doi.org/10.5902/1984644461431

\section{ABSTRACT}

This article presents a preliminary analysis of the Quebec scholarly literature on humanities and social sciences instruction in elementary school published since the 1964 Report of the Royal Commission on Learning. To this end, 83 documents published over roughly the past fifty years were reviewed using a mixed thematic content analysis based on the questions and the conceptual framework underlying the author's previous research. The analysis highlights the trends, perspectives, tensions and breaks characterizing the foundations and conceptual framework of didactics in elementary school social sciences and humanities within the province. Influenced at times by "personalist" and "technological" theories, and at others by constructivist (or even socio-constructivist) ones, this field's evolution bears witness to a diversity of conceptions and viewpoints directly connected to the conceptual basis of those who have collectively and historically shaped it.

Keywords: Didactics; Social sciences and humanities; Elementary school; Quebec.

\section{RESUMO}

O artigo apresenta uma análise preliminar da literatura científica sobre o ensino das ciências humanas e sociais no ensino fundamenta (primário) publicada no Québec após a difusão do Relatório da Comissão Real de Educação em 1964. Para tanto, 83 documentos publicados ao longo dos últimos sessenta anos foram revisados por meio de uma análise temática mista de conteúdo com base nos questionamentos e no quadro conceitual que sustentam as pesquisas anteriores do autor do texto. Destaca, assim, as tendências, perspectivas, tensões e pontos de ruptura no que tange aos fundamentos e à estrutura conceitual específicos ao campo da didática das ciências humanas e sociais no Québec. Ora influenciada pelas chamadas teorias personalistas e tecnológicas, ora pela chamadas teorias construtivistas (e mesmo socioconstrutivistas), a evolução desse campo testemunha da diversidade de concepções e pontos de vista que são diretamente relacionados aos fundamentos subjacentes do pensamento daqueles que a implementam coletiva e historicamente.

Palavras-chave: Didática; Ciências humanas e sociais; Primário; Quebec.

\section{Introduction}

À l'aube des années 1960, le Québec a un rattrapage à faire sur plusieurs sociétés industrielles, notamment en matière d'éducation (LENOIR; LAFOREST, 1995). II faudra, cependant, attendre la prise du pouvoir par le Parti libéral de Jean Lesage pour que la situation commence à évoluer. Plongée dans une ère 


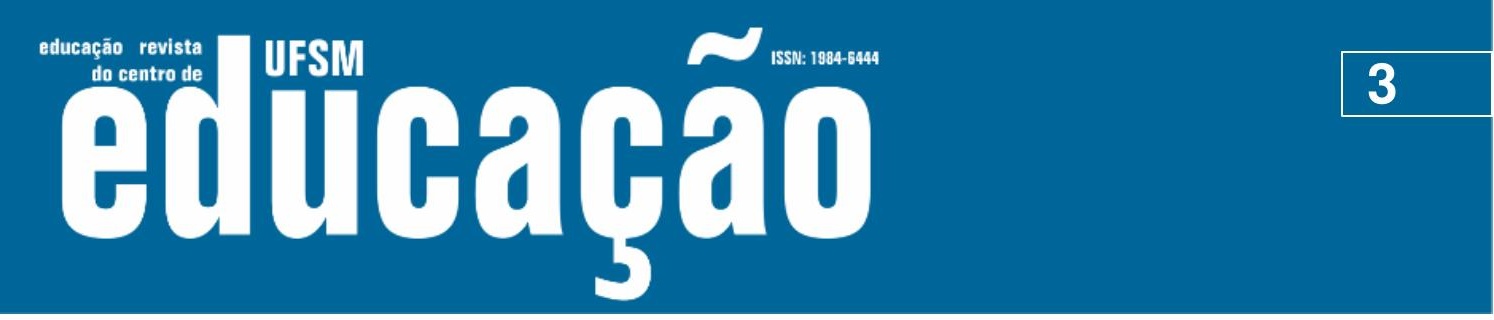

ISSN: 1984-6444 | http://dx.doi.org/10.5902/1984644461431

d'industrialisation de l'économie, d'urbanisation des populations agricoles et de modernisation de ses structures sociales, la société québécoise connaîtra une période de transformations profondes (CHARLAND, 2005; DUFOUR, 1997). Sur le plan éducatif, la prise du pouvoir par le Parti libéral marque le début d'une période de grandes réformes éducationnelles. Dès son ascension au pouvoir, le premier ministre adopte une dizaine de lois connues sous le nom de Grande Charte de l'éducation qui viennent changer radicalement le scénario éducationnel. La plus connue demeure toutefois l'institution de la Commission royale d'enquête sur l'enseignement - Commission Parent - qui avait pour mandat de faire état de l'organisation et du financement de l'éducation québécoise et d'émettre des recommandations au gouvernement (AUDET, 1971).

Avec la publication du Rapport de la Commission Parent (GOUVERNEMENT DU QUÉBEC, 1963-1965), l'orthodoxie qui normalise l'enseignement des sciences humaines et sociales (SHS) est sérieusement secouée. Ce rapport critique profondément son enseignement, entre autres, l'orientation vers une apologétique religieuse et nationaliste des contenus préconisés, le peu d'objectivité dans l'enseignement de l'histoire, les différentes perspectives existantes au sein de l'enseignement de cette discipline entre les commissions scolaires protestantes et catholiques, l'approche pédagogique privilégiée et la formation insuffisante des enseignants (DUPUIS, 1977, 1979; DUPUIS; LAFOREST, 1983; LAFOREST, 1989). Ces critiques associées à la mise en place d'une nouvelle structure d'enseignement favoriseront l'émergence d'une nouvelle vision de l'éducation (LAFOREST, 1989; LENOIR; LAFOREST, 1995) et, par conséquent, d'une nouvelle vision de l'enseignement des sciences humaines et sociales.

En effet, si avant les années 1960 l'enseignement des sciences humaines et sociales $^{1}$ allait de soi et que sa remise en question s'avérait difficile, les critiques soulevées par ce rapport viennent donner un nouveau souffle à la réflexion sur l'éducation et, à la suite de sa parution, de nombreuses publications vont remettre en question la façon traditionnelle de penser l'enseignement des sciences humaines et sociales. Elles proposeront, de surcroît, de nouveaux regards sur les conditions d'enseignement et d'apprentissage des SHS au primaire (DUPUIS; LAFOREST, 1983; 


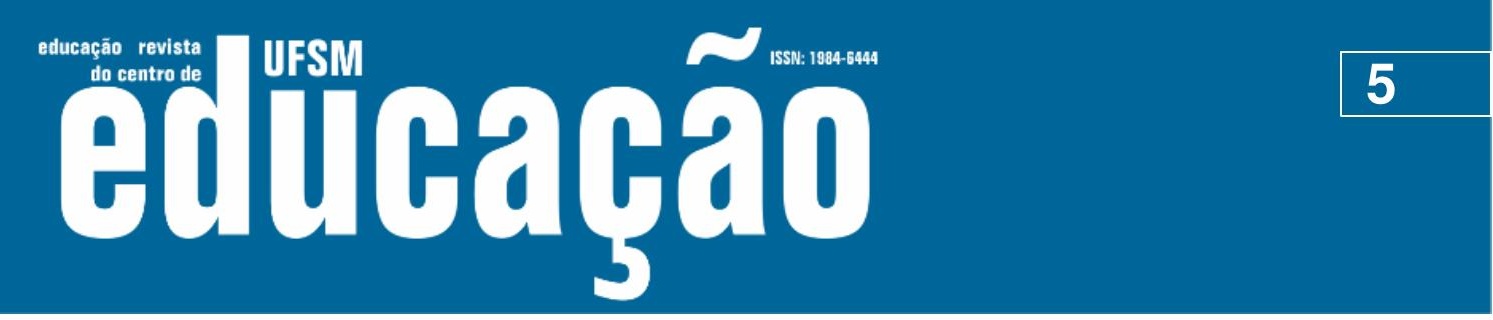

ISSN: 1984-6444 | http://dx.doi.org/10.5902/1984644461431

\section{Vue globale de la documentation scientifique et professionnelle analysée}

Le corpus qui a fait l'objet de la présente analyse est composé de 83 documents ( 50 articles de revues, 19 chapitres de livres, 6 livres, 3 thèses de doctorat, 1 mémoire de maîtrise, 1 rapport de recherche et 3 textes tirés d'actes de colloque) publiés depuis près d'une soixantaine d'années. Faute de banques de données couvrant toute la période sélectionnée, nous avons directement procédé à une recherche dans les principales revues québécoises du domaine des sciences de l'éducation et selon les principaux auteurs identifiés. Trente-cinq documents publiés entre 1964 et 1979 (dans le contexte d'émergence des programmes-cadres), 18 entre 1980 et 1990 (dans le contexte d'émergence des programmes par objectifs) et 30 documents parus après 1991 (dans le contexte d'émergence des programmes par compétences).

La collecte des données a été effectuée par l'entremise d'une analyse thématique mixte de contenu en fonction des questions soulevées précédemment et du cadre conceptuel à la base des travaux de recherche antérieurs réalisés par le chercheur (ARAÚJO-OLIVEIRA, 2015, ARAÚJO-OLIVEIRA; LENOIR; LEBRUN, 2016). La documentation scientifique et professionnelle québécoise portant sur l'enseignement des sciences humaines et sociales a été ainsi analysée selon les aspects suivants qui émergent des questions soulevées: conception des finalités éducatives associées aux SHS, conception des objets d'apprentissage, conception de la didactique, conception du rapport au savoir, conception des modalités d'accession au savoir, conception de la démarche d'apprentissage et conception du rôle de l'enseignant et de l'élève.

Le tableau 1 ci-après présente une vue globale et synthétique de l'analyse réalisée en mettant en relief d'une part la définition des catégories d'analyse et d'autre part les éléments les plus significatifs pour chacun des aspects analysés. Ces éléments seront discutés par la suite. 


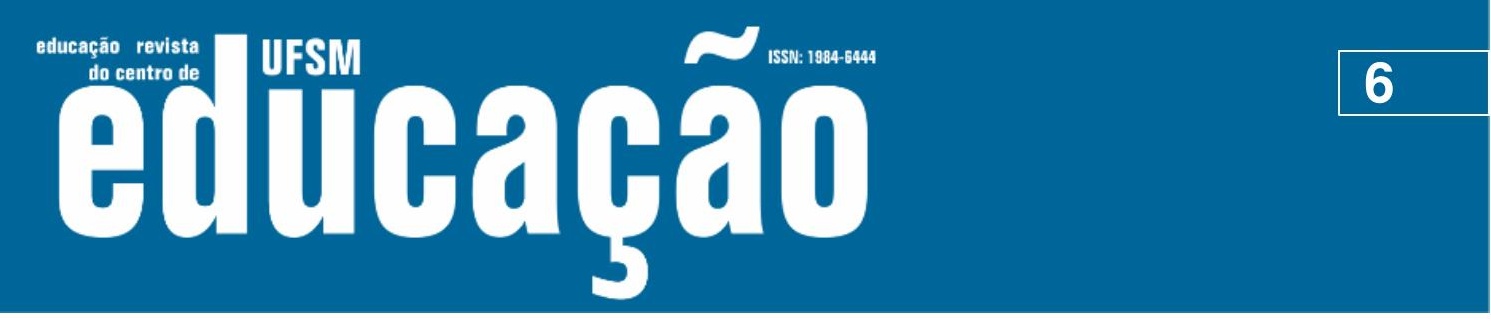

ISSN: 1984-6444 | http://dx.doi.org/10.5902/1984644461431

Tableau 1 - Synthèse des éléments analysés

\begin{tabular}{|c|c|c|c|}
\hline \multicolumn{2}{|c|}{ Éléments analysés } & Catégories & $\mathbf{N}$ \\
\hline \multirow{3}{*}{$\begin{array}{l}\text { Pourquoi } \\
\text { enseigne-t-on les } \\
\text { SHS? }\end{array}$} & \multirow{3}{*}{$\begin{array}{l}\text { Finalités } \\
\text { éducatives }\end{array}$} & $\begin{array}{l}\text { - Finalité adaptative (développement d'attitudes et } \\
\text { valeurs acceptables pour s'adapter à la société } \\
\text { d'appartenance) }\end{array}$ & 20 \\
\hline & & $\begin{array}{l}\text { - Finalité cognitive (développement conceptuel, } \\
\text { habiletés et attitudes intellectuelles, pensée } \\
\text { critique) }\end{array}$ & 12 \\
\hline & & - Finalité utilitaire (développement d'habiletés utiles) & 9 \\
\hline \multirow{6}{*}{$\begin{array}{l}\text { Qu'enseigne-t-on } \\
\text { en SHS? }\end{array}$} & \multirow{6}{*}{$\begin{array}{c}\text { Objets } \\
\text { d'apprentissage }\end{array}$} & $\begin{array}{l}\text { - Cadre de travail spécifique à la discipline } \\
\text { (méthodes, l'observation, la description, attitude } \\
\text { scientifique, etc.) }\end{array}$ & 15 \\
\hline & & $\begin{array}{l}\text { - Habiletés techniques (lecture des cartes, } \\
\text { interprétation de graphiques, etc.) }\end{array}$ & 6 \\
\hline & & $\begin{array}{l}\text { - Données factuelles (personnages, dates, lieux, } \\
\text { événements historiques, etc.) }\end{array}$ & 3 \\
\hline & & $\begin{array}{l}\text { - Habiletés intellectuelles (raisonnement, } \\
\text { argumentation, compréhension, etc.) }\end{array}$ & 7 \\
\hline & & - Concepts, notions et trames conceptuelles & 7 \\
\hline & & - Démarche à caractère scientifique (non naturelle) & 4 \\
\hline \multirow{9}{*}{$\begin{array}{l}\text { À partir de quoi } \\
\text { enseigne-t-on les } \\
\text { SHS? }\end{array}$} & \multirow{3}{*}{$\begin{array}{l}\text { Définition de la } \\
\text { didactique }\end{array}$} & $\begin{array}{l}\text { - Perspective techno-instrumentale (partie de la } \\
\text { pédagogie qui traite des contenus, méthodes et } \\
\text { procédés d'enseignement) }\end{array}$ & 2 \\
\hline & & $\begin{array}{l}\text { - Perspective psychologique (partie de la pédagogie } \\
\text { qui traite des notions que l'élève peut acquérir selon } \\
\text { son niveau de maturité) }\end{array}$ & 1 \\
\hline & & $\begin{array}{l}\text { - Perspective praxéologique (discipline qui traite des } \\
\text { conditions d'enseignement-apprentissage d'une } \\
\text { connaissance spécifique) }\end{array}$ & 1 \\
\hline & \multirow{2}{*}{$\begin{array}{l}\text { Conception du } \\
\text { savoir }\end{array}$} & $\begin{array}{l}\text { - Donné préexistant à l'individu (vérité univoque } \\
\text { existante indépendamment du sujet) }\end{array}$ & 16 \\
\hline & & $\begin{array}{l}\text { - Construit humain (produit de l'action humaine, } \\
\text { socialement et spatiotemporellement déterminé) }\end{array}$ & 10 \\
\hline & \multirow{4}{*}{$\begin{array}{c}\text { Modalités } \\
\text { d'accession au } \\
\text { savoir }\end{array}$} & $\begin{array}{l}\text { - Par révélation (transmission-réception d'un savoir } \\
\text { préexistant) }\end{array}$ & 0 \\
\hline & & $\begin{array}{l}\text { - Par découverte personnelle (observations } \\
\text { systématiques du réel, tâtonnement empirique) }\end{array}$ & 19 \\
\hline & & $\begin{array}{l}\text { - Par dévoilement progressif (découverte basée sur } \\
\text { le principe du stimuli-réponse d'un savoir qui était } \\
\text { déjà là) }\end{array}$ & 5 \\
\hline & & $\begin{array}{l}\text { - Par une activité de construction (processus } \\
\text { d'objectivation dans une rupture avec le réel) }\end{array}$ & 10 \\
\hline \multirow{3}{*}{$\begin{array}{l}\text { Comment } \\
\text { enseigne-t-on les } \\
\text { SH? }\end{array}$} & \multirow{3}{*}{$\begin{array}{l}\text { Démarche } \\
\text { d'enseignement- } \\
\text { apprentissage }\end{array}$} & $\begin{array}{l}\text { - Démarche inductive (observation spontanée, } \\
\text { analyse, communication) }\end{array}$ & 12 \\
\hline & & $\begin{array}{l}\text { - Démarche hypothético-déductive } \\
\text { (problématisation, hypothèse, vérification) }\end{array}$ & 3 \\
\hline & & $\begin{array}{l}\text { - Démarche de conceptualisation (problématisation, } \\
\text { recueil de données, traitement et synthèse) }\end{array}$ & 9 \\
\hline \multirow{2}{*}{$\begin{array}{l}\text { Selon quelle vision } \\
\text { du rôle des } \\
\text { acteurs? }\end{array}$} & \multirow[t]{2}{*}{$\begin{array}{l}\text { Rôle de } \\
\text { l'enseignant }\end{array}$} & $\begin{array}{l}\text { - Animateur, facilitateur, guide (suscite l'intérêt de } \\
\text { l'élève pour la découverte par la création d'un climat } \\
\text { favorable) }\end{array}$ & 7 \\
\hline & & $\begin{array}{l}\text { - Programmeur (structure l'apprentissage selon un } \\
\text { ordre logique, le chemin que doit suivre l'élève) }\end{array}$ & 3 \\
\hline
\end{tabular}




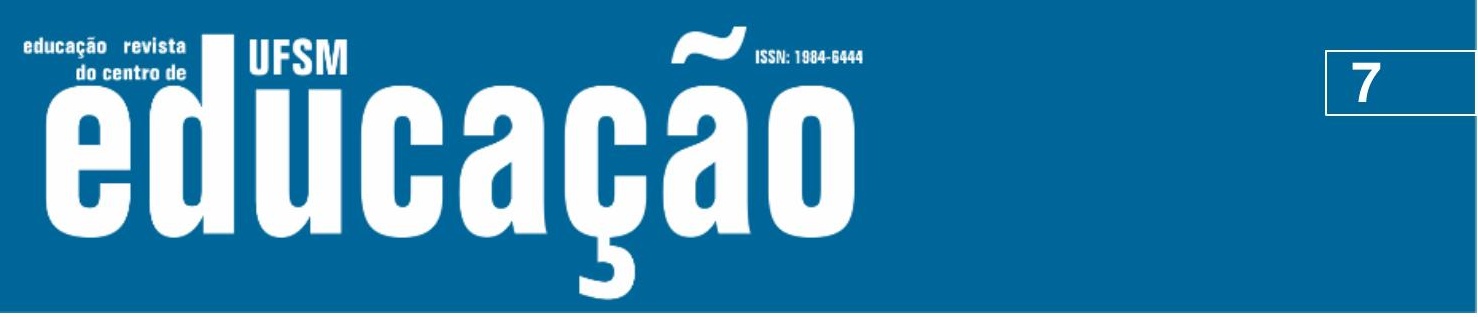

ISSN: 1984-6444 | http://dx.doi.org/10.5902/1984644461431

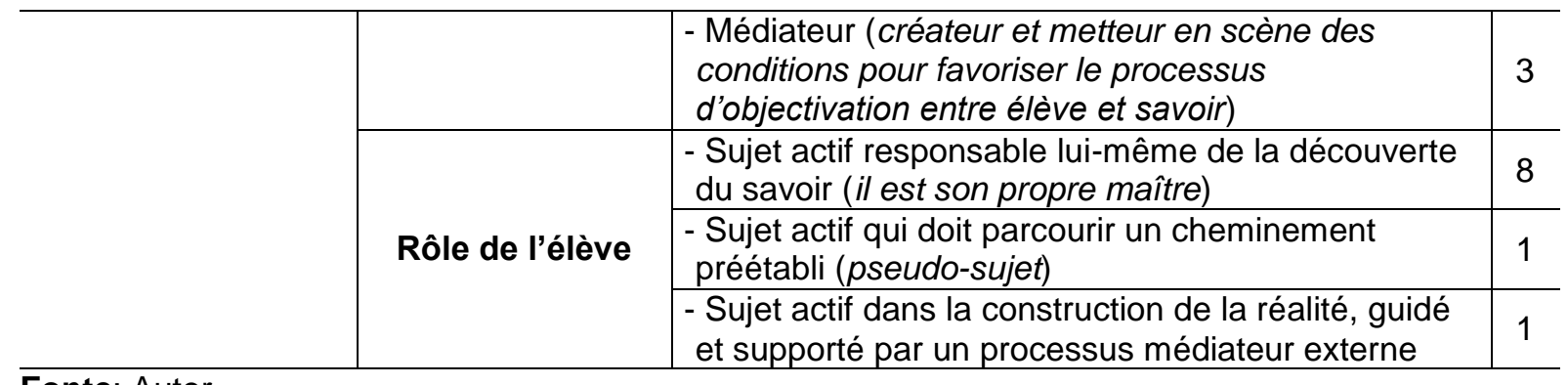

Fonte: Autor

\section{Tendances, perspectives, tensions et points de ruptures sous- jacentes à la documentation analysée}

L'analyse de la documentation scientifique et professionnelle présentée synthétiquement dans la section précédente a permis de faire émerger quelques tendances, perspectives, tensions et points de rupture qui seront ici présentés principalement par l'entremise de citations jugées pertinentes pour la compréhension de la pensée des différents auteurs consultés.

\section{Pourquoi enseigne-t-on les sciences humaines et sociales au primaire (conception des finalités éducatives associées aux SHS)?}

Sur le plan des finalités associées à l'enseignement des sciences humaines et sociales, on constate une structure constituée selon trois finalités principales: finalités adaptatives (développement d'attitudes et valeurs acceptables pour s'adapter à la société d'appartenance); finalités cognitives (développement conceptuel, habiletés et attitudes intellectuelles, pensée critique) et finalités utilitaires (développement d'habiletés utiles).

Dans le cas d'une vingtaine de documents analysés, les SHS, par l'apprentissage du comment vivre ensemble et par la formation de la personne qui lui est traditionnellement associée (LAURIN, 1998), doivent permettre à l'élève l'acquisition d'attitudes plutôt que de connaissances (DUPUIS; LAFOREST, 1972). Elles doivent également l'amener à prendre connaissance de la réalité immédiate qui l'entoure (BOISSEAU, 1977; PICARD, 1977) et en conséquence à mieux s'adapter à 


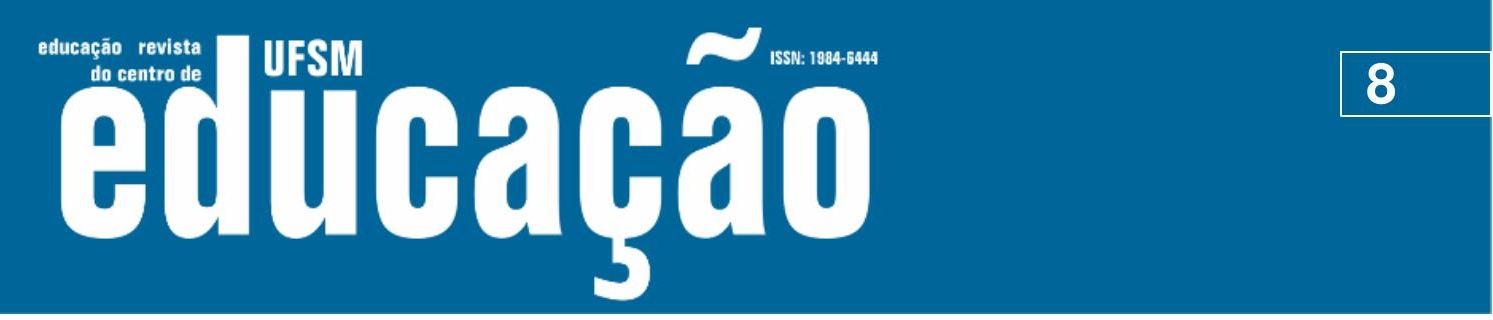

ISSN: 1984-6444 | http://dx.doi.org/10.5902/1984644461431

la société et à ses changements continuels (GÉLINAS, 1976), à s'ouvrir au monde et aux autres cultures, à développer un esprit démocratique (LEBRUN, 1993), etc. En SHS, affirme Robert (1983), la connaissance ne saurait se dissocier de l'expression des valeurs individuelles et collectives.

La finalité dite cognitive arrive au second rang comme facteur favorisant le développement intellectuel. Les documents qui s'inscrivent sous une telle rubrique soutiennent que la principale raison d'être de l'enseignement des sciences humaines et sociales est de permettre à l'élève de conceptualiser et de structurer sa représentation du monde à partir de l'étude de divers concepts, notions et trames conceptuelles (LEBRUN, 1993; LEBRUN; LENOIR, 2001; LENOIR, 1989) et, de ce fait de développer sa pensée critique et réflexive (DUPUIS; LAFOREST, 1983; LAURIN, 1999; LAVILLE, 1991; LEBRUN, 1993).

Une troisième finalité se démarque par le caractère utilitariste attribué à l'enseignement des sciences humaines et sociales. II s'agit des documents dont les auteurs considèrent que la finalité primordiale de l'enseignement des sciences humaines et sociales est de favoriser l'acquisition d'habiletés utiles à l'élève (ex.: CHOQUETTE, 1980; MARTINEAU, 1988; PICARD, 1977; ROBERT, 1983), c'est-àdire des habiletés qui lui serviront régulièrement dans la vie de tous les jours (ex.: l'emploi de cartes, interprétation de graphiques, lecture d'une ligne de temps, etc.).

\section{Qu'enseigne-t-on en sciences humaines et sociales au primaire (conception des objets d'apprentissage et d'évaluation)?}

En lien étroit avec les finalités mises en avant, six éléments ont été identifiés dans la documentation analysée comme étant au cœur des apprentissages visés en SHS. Tout d'abord, en refusant massivement toute prescription de contenus, sous prétexte qu'« il faut se débarrasser des programmes détaillés comme des Tables de la Loi » (LEFEBVRE, 1978, p. 126), l'objet d'apprentissage visé en SHS doit être, pour 15 documents analysés, un cadre de travail spécifique aux SHS. Ce cadre devrait permettre l'acquisition d'attitudes plutôt que de connaissances (DUPUIS; LAFOREST, 1972), car, précise Gingras (1973), les connaissances importent très peu. L'essentiel 


\title{
T usm Autfarato

ISSN: 1984-6444 | http://dx.doi.org/10.5902/1984644461431

respectivement. Néanmoins, il faut préciser que ces deux éléments sont nécessairement liés au développement conceptuel et aux habiletés intellectuelles. D'une part, le développement conceptuel requiert le recours de la part de l'élève à une démarche d'apprentissage peu naturelle qui doit, elle aussi, faire l'objet d'un apprentissage (LENOIR; LAFOREST, 1994). L'acquisition de ces démarches, selon Lebrun et al. (2008), est la capacité de mise en perspective et d'application d'un raisonnement rigoureux et systématique qui est au cœur de l'enseignementapprentissage en SHS, c'est-à-dire la pensée critique (LAURIN, 1999). D'autre part, ce développement conceptuel ne saurait être effectif en mettant de côté ces repères indispensables pour se situer dans le temps et dans l'espace qui sont les données factuelles (LEBRUN, 1993; LEBRUN; LENOIR, 2001).

\section{À partir de quoi enseigne-t-on les sciences humaines au primaire (conception de la didactique, conception du rapport au savoir, conceptions et modalités d'accès au savoir)?}

Seulement trois documents analysés définissent explicitement la didactique. D'un côté, au début de la période analysée, la didactique est assimilée à la pédagogie:

\begin{abstract}
science auxiliaire de la pédagogie à laquelle cette dernière délègue, pour la réalisation du détail, des tâches éducatives plus générales. Comment amener l'enfant à acquérir telle notion, telle opération ou telle technique de travail? [...] quelles sont les notions qu'un enfant peut acquérir selon son niveau de maturité intellectuelle. (LACHANCE, 1965, P. 65);

ou encore à la partie de la pédagogie qui traite des méthodes et procédés d'enseignement, par opposition à celle qui traite de l'éducation au sens large (SAUCIER, 1968).
\end{abstract}

D'un autre côté, à la toute fin de la période analysée, la didactique est définie en tant que discipline dont les frontières seraient bien délimitées. Ainsi, Lebrun et al. (no prelo), en s'appuyant sur Tochon (1999), conçoivent la didactique comme suit: « A disciplinary didactics describes and designs the actualized or virtual learning and teaching relationships among a disciplinary content, a learner (or learners) and a teacher » (TOCHON, 1999 dans LEBRUN et al,. no prelo). Ils soulignent également, en citant Balacheff et Laborde (1992), que « la recherche en didactique a pour principal 


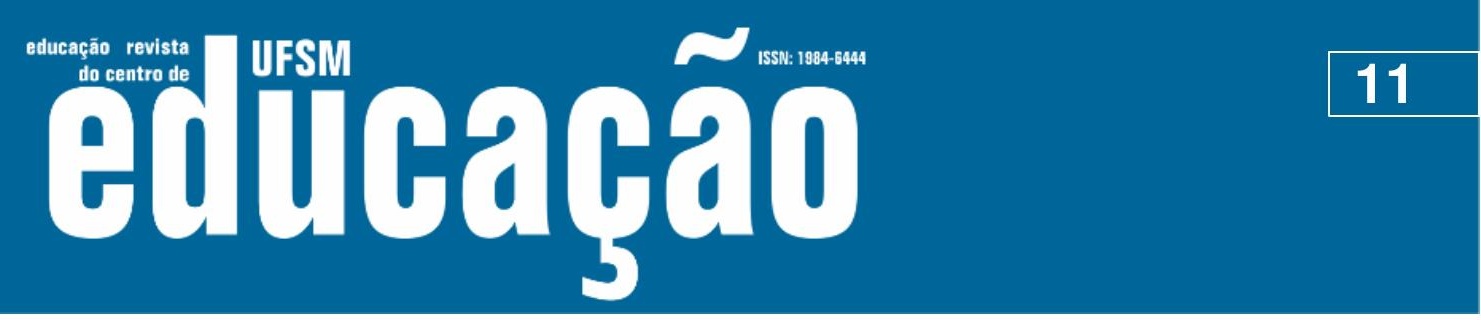

ISSN: 1984-6444 | http://dx.doi.org/10.5902/1984644461431

objet d'étude des conditions de la construction des connaissances complexes par des sujets humains [...] dans des situations délibérément organisées à cette fin [...]. L'originalité de ces recherches réside dans la prise en compte, tant au niveau méthodologique que théorique, de la spécificité des connaissances en jeu » (BALACHEFF; LABORDE, 1992 dans LEBRUN et al., no prelo).

Cette dernière définition se distingue des précédentes, notamment parce qu'elle met l'accent sur le fait qu'il existe des points communs et des caractères spécifiques à chacune des disciplines enseignées, mais aussi sur des aspects essentiels de l'intervention didactique.

En ce qui a trait au rapport au savoir (conceptions et modalités d'accès au savoir), nous avons repéré d'un côté les conceptions de ce qu'est le savoir et, de l'autre, les conceptions des modalités d'accession à celui-ci. Une première conception du savoir, identifiée notamment dans la documentation qui date d'avant les années 1980, renvoie à l'idée selon laquelle le savoir constitue un donné préexistant au sujet humain et indépendant de son existence et de son action. Seize documents laissent entrevoir une telle conception. Ainsi, pour Gélinas (1976, p. 36), « pour bien voir, il suffit d'enlever les œillères, de lever ses toiles et de regarder. Car c'est avec un regard attentif que les questions surgissent rapidement et que l'on prend conscience que nous vivons dans un monde plein de réalités qui ne demandent qu'à être connues ". L'idée sous-jacente est celle d'un savoir donné a priori. II ne s'agit que de les connaître par un contact direct avec la réalité immédiate. Ainsi, « à partir de son expérience à lui, expérience constamment enrichie et contrôlée par l'observation, l'enfant s'appliquera à pénétrer la complexité de l'homme et de la société » (LEFEBVRE, 1964, p. 68). C'est donc par le contact direct avec la réalité que l'élève pourra se faire " une idée aussi juste que possible de l'homme »(LEFEBVRE, 1964, p. 68) et des rapports existants entre l'individu et la société ainsi que des rapports entre différentes sociétés. Cette conception, toutefois, est loin d'être partagée à l'unanimité par l'ensemble des auteurs dans la documentation analysée. Pour Lebrun et Lenoir (2001) et Spallanzani et al. (2001), la réalité est le produit de l'action humaine, celle-ci étant déterminée socialement et spatiotemporellement. Elle ne procède aucunement d'un donné préexistant. Dans ce sens, le savoir « constitue une réponse spécifique à un 


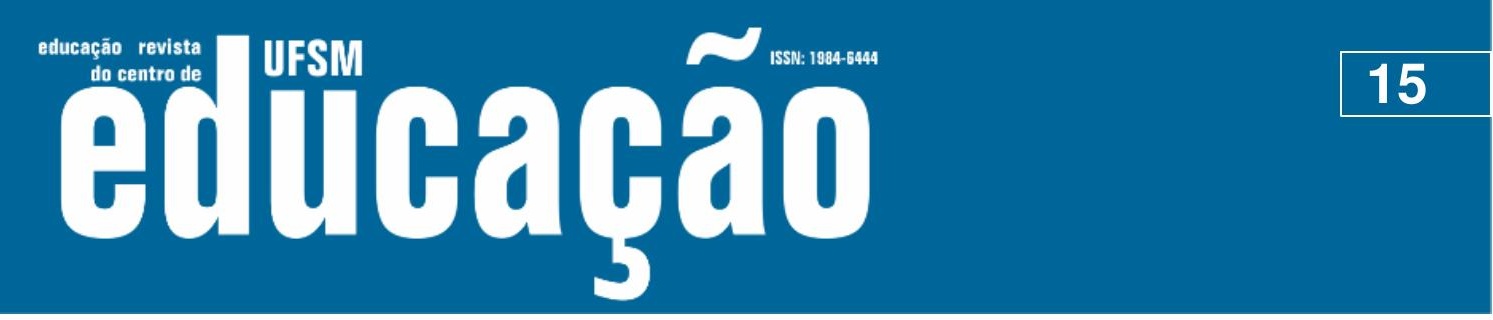

ISSN: 1984-6444 | http://dx.doi.org/10.5902/1984644461431

1) une phase d'investigation spontanée qui, par une situation problème ancrée dans la réalité individuelle ou sociale de l'enfant, a pour but de soulever un questionnement permettant d'explorer et d'exprimer ses perceptions initiales et conduit à la formulation de questions de recherche;

2) une phase d'investigation structurée au sein de laquelle l'enfant soutenu, guidé et encadré par l'enseignant, élabore un plan de recherche qui inclut l'identification des informations à obtenir, l'élaboration de l'outil de collecte et la collecte de données;

3) une phase de structuration régulée dans laquelle les données recueillies font l'objet d'une organisation, d'une classification, d'une mise en relation et d'une production synthèse de manière à permettre la (re)structuration du système d'interprétation du réel. Cette nouvelle compréhension du monde est finalement objectivée au regard des perceptions initiales exprimées en début de séquence et au regard de la démarche utilisée.

\section{Selon quelle vision du rôle des acteurs impliqués dans le processus d'enseignement et d'apprentissage (conception du rôle et de la place de l'enseignant et de l'élève) enseigne-t-on les SHS?}

Seulement 13 documents font explicitement référence au rôle de l'enseignant alors que 10 mentionnent celui de l'élève. Pour Allard (1971), le rôle de l'enseignant est comparable à celui de l'instructeur de baseball. Son rôle n'est pas de jouer à la place de l'élève, mais plutôt de le mettre en activité. II s'agit de celui qui suscite la motivation et l'intérêt de l'élève à la découverte du monde (LAUZON, 1965). On parle alors d'un animateur, d'un facilitateur, d'un guide qui a pour fonction de créer un climat ou un esprit favorable à l'épanouissement de l'enfant (GÉLINAS, 1976; JOHNSON, 1973; LAMARCHE, 1980; LEFEBVRE, 1976, 1978). Ainsi, en lien avec cet enseignant « témoin » des découvertes de l'enfant, le rôle de l'élève ne peut être pensé qu'en tant que sujet actif et responsable lui-même de cette découverte. L'élève devient le centre des apprentissages (ALLARD, 1971; JOHNSON, 1976B) dont il est le seul responsable en devenant lui-même son propre maître (JOHNSON, 1974b). 


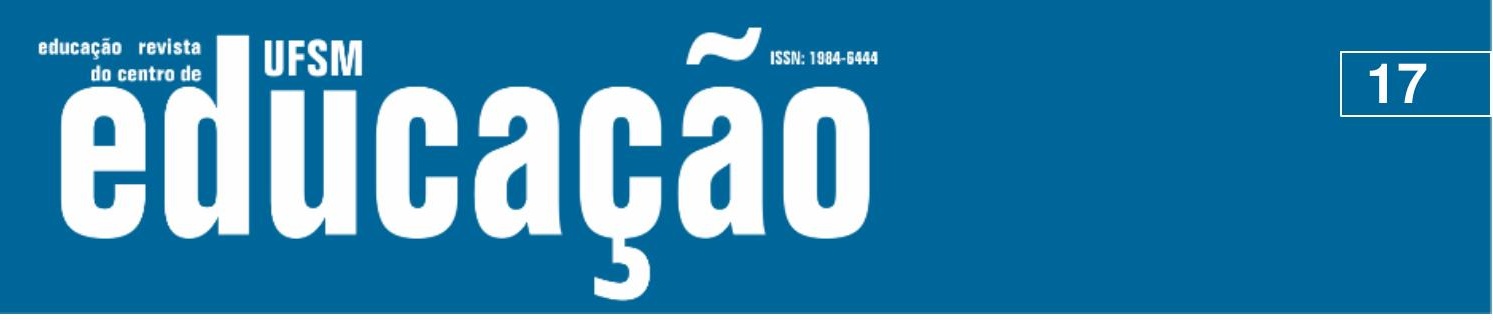

ISSN: 1984-6444 | http://dx.doi.org/10.5902/1984644461431

a mis en évidence quelques tensions et ruptures concernant les fondements, les perspectives et la trame conceptuelle propres au champ de la didactique des SHS au primaire au Québec après la parution de ce rapport:

- Des tensions entre une perspective identitaire ancrée sur une vision descriptive (finalité de socialisation) et une vision critico-réflexive ancrée sur la formation de la pensée critique (finalité d'émancipation);

- Des tensions entre une didactique centrée sur une orientation psychologique et instrumentale (sujet qui sait s'adapter à sa société d'appartenance) et une orientation praxéologique (sujet qui sait réfléchir et agir en conséquence);

- Des tensions entre un savoir réifié (donné et extérieur) et un savoir construit par le sujet apprenant (vision plus constructiviste);

- Des tensions entre des démarches dites naturelles et des démarches à caractère scientifique.

Voilà quelques éléments qui caractérisent ce champ et qui feront l'objet d'une analyse plus approfondie dans le cadre d'un second article (ARAÚJO-OLIVEIRA, no prelo). Influencées tantôt par des théories dites personnalistes, et des théories dites technologiques (néobehavioristes), tantôt par des théories dites constructivistes (voire socioconstructivistes) (BERTRAND, 1998), les tensions identifiées témoignent d'une diversité de conceptions et de points de vue. Ces derniers sont directement liés aux fondements sous-jacents à la pensée de ceux qui collectivement et historiquement la mettent en œuvre et nous invitent, chercheurs en éducation et formateurs d'enseignants, à faire preuve d'une grande prudence quant à la transposition de certains concepts, méthodes et perspectives, que ce soit du champ de la psychologie, de la sociologie, de la formation, de l'économie et même de la discipline scientifique dont cette didactique se veut la représentante.

\section{Références bibliographiques}

ALLARD, Michel. Le rôle de l'enseignant. Courrier pédagogique québécois, Québec, n. 3, v. 2, p. 49-51, 1971.

ALLARD, Michel. Approche pédocentrique et contenu. Courrier pédagogique québécois, Québec, n. 4, v. 2, p. 92-93, 1972. 


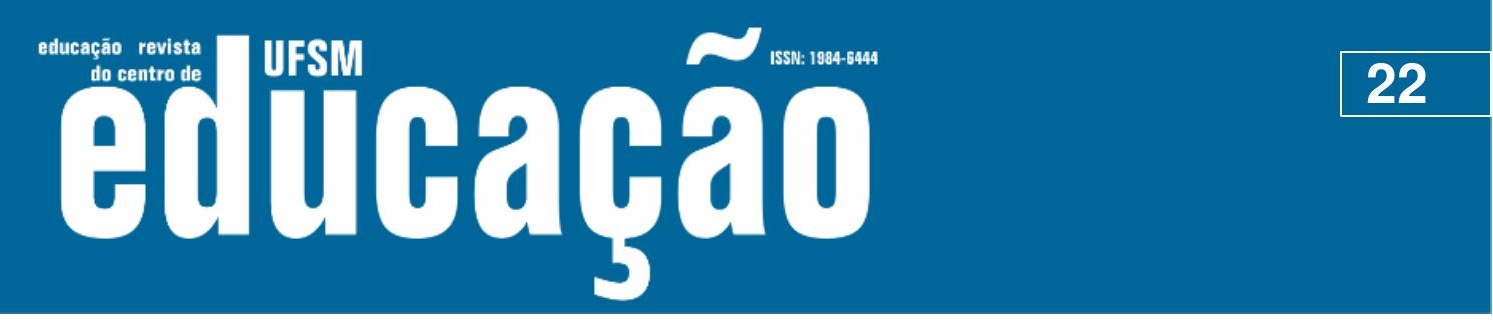

ISSN: 1984-6444 | http://dx.doi.org/10.5902/1984644461431

MARTINEAU, Robert. Pourquoi élaborer des programmes d'histoire par objectifs? Traces, n. 26, v. 5, p. 13-14, Québec, 1988.

PICARD, Jean-Luc. Les sciences humaines d'ici ou d'ailleurs? Bulletin de liaison de la société des professeurs d'histoire du Québec, n. 15, v. 2, p. 8-9, Québec, 1977.

ROBERT, B. L'enseignement de la géographie: Les nouveaux programmes de géographie pour l'enseignement secondaire au Québec. Cahiers de géographie du Québec, n. 27, v. 72, p. 453-459, Québec, 1983.

SAUCIER, R. Définition du mot didactique. Bulletin de liaison de la société des professeurs d'histoire du Québec, n. 1, v. 3, p. 10-11, Québec, 1968.

SPALLANZANI, Carlo. et al. Le rôle du manuel scolaire dans les pratiques enseignantes au primaire. Sherbrooke: Éditions du CRP, 2001.

TREMBLAY-DESROCHERS, M. La problématique du développement des habiletés intellectuelles dans l'enseignement des sciences humaines au primaire. In: LENOIR, Yves; LAFOREST, Mario (Org.). L'enseignement des sciences humaines au primaire: Développement, sous-développement ou développement du sousdéveloppement?. Sherbrooke: Éditions du CRP, 1991, p. 111-120.

\section{(@) $(1) \Theta$}

This work is licensed under a Creative Commons Attribution-NonCommercial 4.0 International (CC BY-NC 4.0)

\section{Notas}

\footnotetext{
${ }^{1}$ L'enseignement des sciences humaines et sociales au primaire visait, avant les années 1960, la formation du chrétien et du patriote par le biais de l'inculcation de connaissance, d'habiletés, d'attitudes et de valeurs pré-identifiées (DUPUIS, 1977, 1979; DUPUIS; LAFOREST, 1983; LAFOREST, 1989).

2 Dans ce texte, nous entendons par champ de la didactique des SHS toute réflexion ou recherche portant sur les conditions d'enseignement et d'apprentissage du point de vue de leur spécificité disciplinaire.

${ }^{3}$ Par documentation scientifique, il faut entendre ici à la fois les publications issues d'une ou de plusieurs recherches (articles de revue scientifique, livres, thèses, etc.) et les publications rédigées par des universitaires à l'intention des acteurs du milieu scolaire ou de la formation à l'enseignement (articles de revue professionnelle, livres et documents divers).
} 